\title{
Network Analysis using GIS Techniques: A Case of Chandigarh City
}

\author{
Parveen Kumar ${ }^{1}$, Dinesh Kumar ${ }^{2}$
}

\begin{abstract}
ArcGIS based Network Analysis provide multiple solution aspects for a variety of problems pertaining to spatial networks include finding the most efficient travel route, generating travel directions, locating the closest facility, and defining service areas based on travel time and distance covered. The present study tries to analyze the potential use of network analysis in defining the optimal service area of different services such as hospitals, schools and fire stations of Chandigarh city. Google earth image of Chandigarh city has been used for this study; it was then geo-referenced. Digitization was carried by using Shape file generated for different analysis. The network analysis tool was used to measure the efficiency of services in terms of time and distance. It also help in analyzing the gap existing in the spatial allocation of these services in city area and whether the existing resource allocation is good or bad in term of efficiency of these services.
\end{abstract}

Keywords: Efficiency, GIS, Network Analysis, Optimal, Services.

\section{Introduction}

Geographical Information System (GIS) is a tool which has been employed for integration of spatial and non-spatial data. GIS is a specific integrated system of hardware, software and procedure designed to support capture, management, manipulation, analysis and display of spatially referenced data for solving complex planning and management problems. GIS based Network Analyst is a powerful extension that provides network-based spatial analysis including routing, travel directions, closest facility, and service area analysis. Using a sophisticated network data model, users can easily build networks from their geographic information system (GIS) data. A country's transportation system represents development stage of country. But at the same time highly developed countries are facing higher problems of transportation management and spending lots money and effort for solving those problems. Growing traffic congestion, the need to preserve the environment, and the problems of road safety are the main reasons for many cities worldwide to consider new initiatives in public transit systems. ArcGIS Network Analyst enables users to dynamically model realistic network conditions, including turn restrictions, speed limits, height restrictions, and traffic conditions, at different times of the day. So it could be of great help for Transportation Engineering and planning also. When so many parameters are to be connected with Transportation network like travel time, speed, road resistance, turning movements, etc. For such a big network GIS proves itself as an efficient tool for solving such a network problems quickly and with a great precision.

\section{Aim \& Objective}

Aim:

Service area based network analysis of Chandigarh city using GIS techniques.

Objective:

- To identify the shortest route between two locations which is less time and less cost consuming

- To define service area based on distance and time for different facilities

\section{Methodology}

\subsection{Study Area}

Chandigarh is a union territory of India and a joint capital for the states of Haryana and Punjab. Its area is $114 \mathrm{sq} . \mathrm{km}$. the city is known as one of the best experiment in modern urban planning as well as for modern architecture in India. It was planned by famous French architecture Le Corbusier. The city has a good network of road system with different hierarchies. A hierarchy of road separated pedestrian and vehicular traffic into seven different road types with their different characteristics. These are following:

- V-1 Fast roads connecting Chandigarh to other towns.

- V-2 Arterial roads.

- V-3 Fast vehicular sector dividing roads.

- V-4 Meandering shopping streets.

- V-5 Sector circulation roads.

- V-6 Access roads to houses

- V-7 Foot paths and cycle tracks

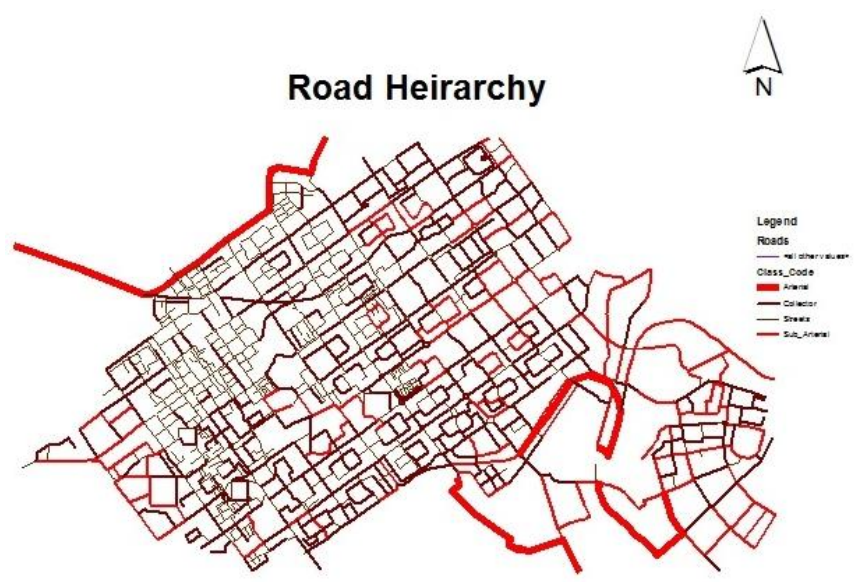

\subsection{Material and Methods}

For the network analysis of the Chandigarh city a methodology was framed which includes following steps: 


\section{International Journal of Science and Research (IJSR) \\ ISSN (Online): 2319-7064}

Index Copernicus Value (2013): 6.14 | Impact Factor (2014): 5.611

1) Data used

A Google earth satellite imagery, which was geo-referenced in ArcGIS, was used for the network analysis of the Chandigarh city. Furthers, point location of public services such as hospitals, schools, colleges and fire station were obtained from Google image and their Shape-file were generated using ArcGIS.

\section{2) Geo-data base Creation}

For generating Geo-database following data has been used.

1. Chandigarh city base map using Google imagery

2. City road network Shape file

3. Shape file of public services such as hospital, schools, colleges and fire station.

3) Data processing and Analysis

For the data processing following steps were taken:

- Geo-referencing of Chandigarh city image

- Generation of Shape file of hospitals, schools, colleges and fire station,

- Digitization of road network

- Generate topology

- Generating Network Geo-dataset

- Analysis by using network analysis tool

\section{Results and Discussion}

Identify the shortest route between two locations which is less time and less cost consuming

By using network analysis tools, shortest route ${ }^{\text {ee }}$ was created between two different locations, which is more efficient in terms of less time and subsequently, cost consumed in travelling. Shortest route analysis finds the route with minimum cumulative impedance between nodes on a network. The route may connect just two nodes - an origin and a destination or have specific stop between these two nodes.

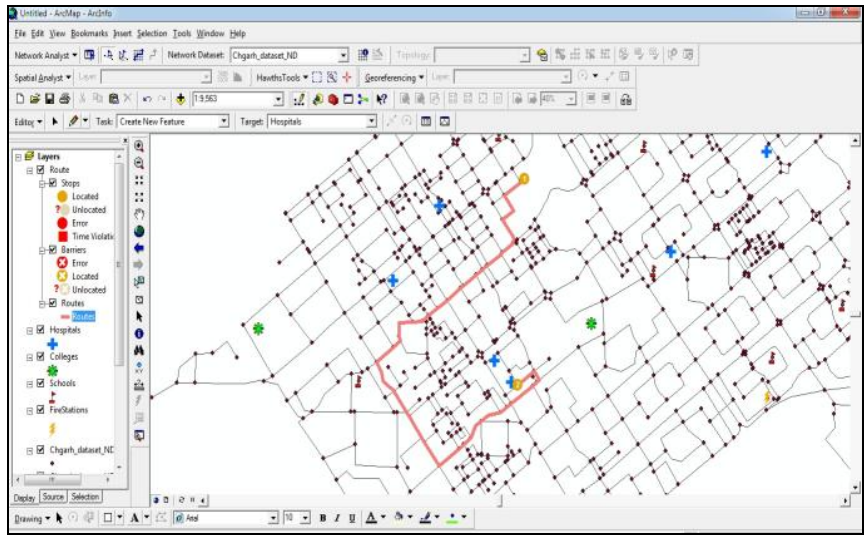

Define service area based on distance and time for different facilities

The map below indicates the service area of different closest facilities based on the distance and time. By this analysis it may be analysed the actual service area of different facility or whether these facilities are enough for that area, if not then how much is required. For the present study, services such as hospitals, schools, fire stations were chosen for analysis purpose and defined their service area based on time and distance.

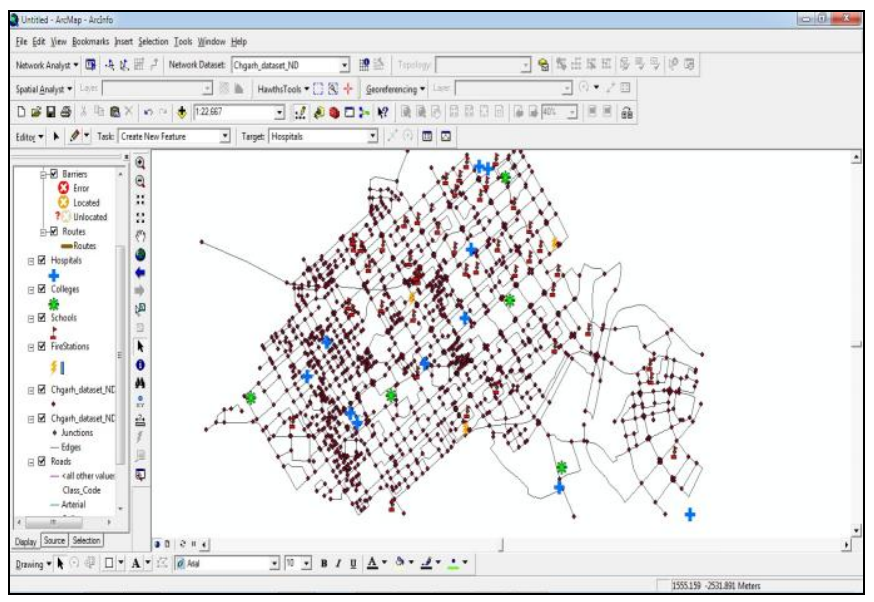

The map above shows the spatial distribution of different services such as hospitals, schools, colleges and fire station through a network. This is also called as service allocation analysis. Because the allocations of these services define the extent of their service area and the main objective of spatial allocation analysis is to measure the efficiency of these services in terms of time and distance.

\section{Service Area for Hospitals}

$\frac{\Delta}{N}$

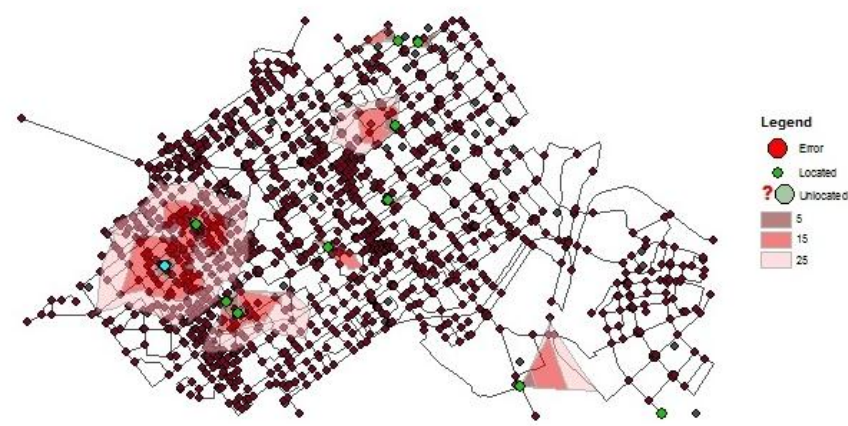

The map above shows the service area of each hospital based on the multiple buffers of estimated travel time of 5, 15, and 25 minutes which indicates the actual service area with efficiency.

Service Area for Hospitals
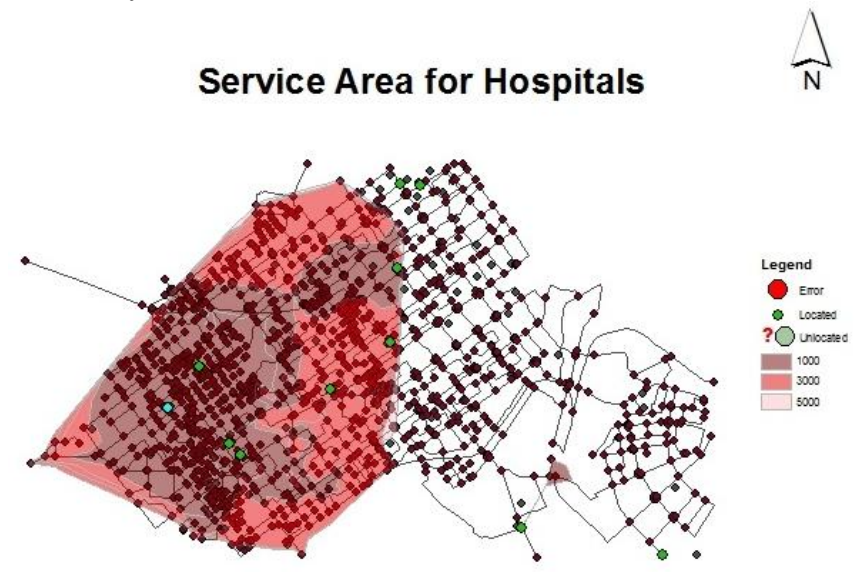


\section{International Journal of Science and Research (IJSR) \\ ISSN (Online): 2319-7064}

Index Copernicus Value (2013): 6.14 | Impact Factor (2014): 5.611

This service area map of hospitals with multiple buffers is based on distance interval of 1000 meters, 3000 meters and 5000 meters showing the actual service area with efficiency.

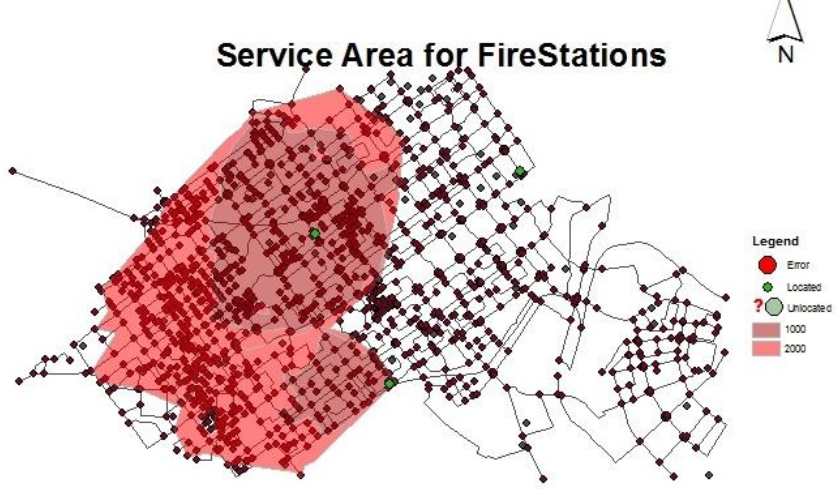

This map shows the service area of fire stations based on distance. With a buffer of 1000 meters which shows the core service area and the 2000 meters buffer indicate the areas which are yet to be served by these fire stations.

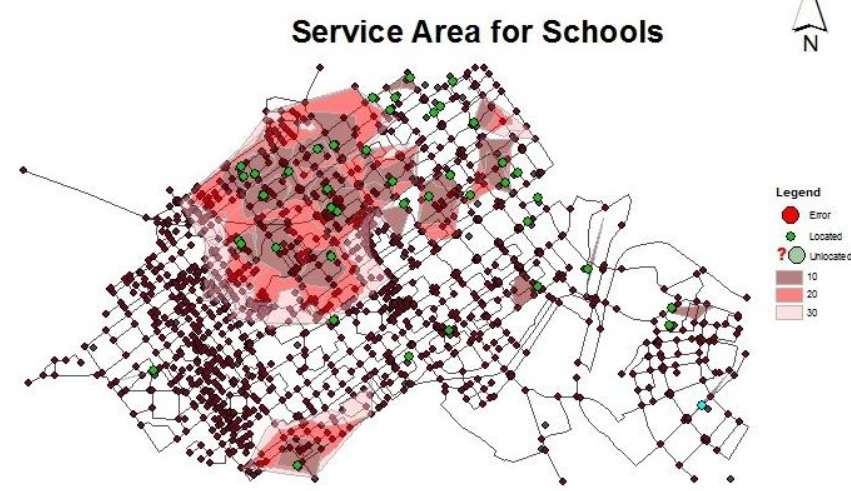

The map above shows the service area of schools based on the average time taken to reach in schools. The service area of schools with a multiple buffers of 10, 20, 30 minutes showing the actual service area with efficiency.

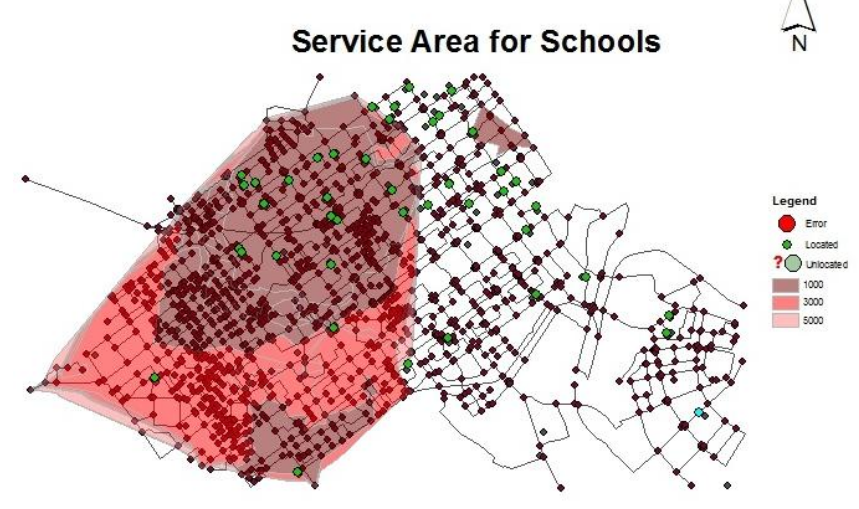

This map above shows the service area of schools based on multiple buffers of 1000/3000/5000 meters with high, moderate and low efficient service area of the school respectively.

\section{Conclusion}

The network analysis tool is most reliable to measure the efficiency of services in terms of time and distance. In the study, optimal service area of services such as hospitals, schools and fore station were defined based on the time and distance. It also help in analyzing the gap exist in the allocation of these services in city area and also help us in analyzing whether the existing resource allocation is good or bad in term of efficiency of these service area. So this study tries to analyze the potential use of GIS tools for network analysis. In fact by using network analysis tools number of things can analyze which are more relevant for different type of network analysis especially for transport planning we can create this type of analysis for different purposes like shortest path analysis, closest facility analysis, service area determined analysis and also for the best recourses allocation and for the creating of emergency route services, so this type of analysis is very crucial especially for the transport based planning.

\section{Acknowledgement}

The authors would like to thank his friends Naveen Kumar, Sayan Roy, Arvind Kaushik for helping at every moment during crafting out the paper work. Finally, the authors would like to thank his parent for the continuous support.

\section{References}

[1] http://chandigarh.gov.in/knowchd general.htm

[2] Facility, Closest, and Service Area Analysis. "ArcGIS Network Analyst."

[3] Fang, Kun, Polygon Based Model, and Xu Yiqin. "Gis Network Analysis in Rescue of Coal Mine.” (2001)

[4] http://help.arcgis.com/en/arcgisdesktop/10.0/pdf/networkanalyst-tutorial.pdf

[5] Smith, Richard C, David L Harkey, and Bobby Harris. "Implementation of GIS-Based Highway Safety Analyses : Bridging the Gap." January (2001)

[6] http://webhelp.esri.com/arcgisdesktop/9.2/pdf/Network_A nalyst_Tutorial.pdf

\section{Author Profile}

Paveen Kumar received the Degree in Rural Planning and Management from CEPT University, Ahmedabad in 2013 and now doing M.Phil in Department of Geography, Kurukshetra University, Kurukshetra, Haryana, India

Dinesh Kumar received the Degree of M.Sc. in Geography from Department of Geography, Kurukshetra University, Kurukshetra, Haryana, India. 